\title{
CULTURA MATERIAL E PARADIGMA PRODUÇÃO-CONSUMO-MEDIAÇÃO: UMA ABORDAGEM METODOLÓGICA INTERDISCIPLINAR PARA PESQUISAS NA ÁREA DA HISTÓRIA DO DESIGN
}

\author{
Amélia Paes Vieira Reis (discente)/UFPE \\ Silvio Romero Barreto Campello (orientador)/UFPE
}

\section{RESUMO}

Este artigo apresenta uma proposta de abordagem metodológica interdisciplinar para pesquisas na área de História do Design que tenham como objeto de estudo artefatos gráficos políticos. Para isso, relacionamos o paradigma Produção-consumo-mediação de Lees-Maffei (2009) a contribuições de autores da Cultura Material (Meneses, 1998 e 2003; Miller, 1998; Woodward, 2007). A partir dos resultados de uma revisão bibliográfica preliminar e de uma Revisão Sistemática da Literatura (RSL), são apresentadas três correntes metodológicas principais do campo da História do Design (Huppatz, 2021; Lees-Maffei, 2009). Argumentamos que a metodologia proposta é particularmente útil para investigar artefatos gráficos políticos dentro de um contexto histórico, do ponto de vista do design. As discussões aqui apresentadas fazem parte da pesquisa de doutorado "Design Político em Pernambuco: artefatos gráficos e a redemocratização do Brasil (1979-1989)", em desenvolvimento no PPGdesign/UFPE, na linha de pesquisa Design da Informação, sob a orientação do prof. Silvio Barreto Campello.

Palavras-chave: artefatos gráficos políticos; história do design - design político - mediação - cultura material. 


\section{Introdução}

Em estudos na área da História do design, é frequente o foco no objeto, seus autores e processos de criação e produção. Esta foi a ênfase das primeiras pesquisas do campo e é predominante até hoje (Huppatz, 2021; Lees-Maffei, 2009).

Entretanto, em se tratando de artefatos gráficos políticos, percebemos que levar a investigação para além do circuito criação/produção e olhar também para a veiculação e uso dentro do jogo político poderia enriquecer a pesquisa. O ponto de partida então, foi buscar uma abordagem metodológica que permitisse um equilíbrio entre a observação da materialidade e especificidade formal e informacional dos artefatos, e a compreensão das relações sujeito-objeto.

Entendemos a dimensão política como vinculada a espaços de troca e diálogo (Mouffe, 2005) e que os processos de criação, produção, veiculação/uso dos artefatos podem ser vistos como uma ação política. Em decorrência, reforçamos o potencial do design em mediar ideologias e comportamentos. Como aponta Markussen (2013, p. 39):

\footnotetext{
“A ação política do design não é um boicote, greve, protesto, demonstração, ou algum outro ato político; em vez disso, ela empresta seu poder de resistência por ser precisamente uma forma projetada de intervir na vida das pessoas".
}

Neste artigo, apresentamos uma proposta de abordagem metodológica interdisciplinar que relaciona o Paradigma Produção-consumo-mediação de Lees-Maffei (2009) e o campo da Cultura Material através de Meneses $(1998,2003)$, Miller (1998) e Woodward (2007). Levamos em conta que o cunho político dos artefatos demanda um olhar analítico tanto para os seus aspectos produtivos, quanto para a sua relação com a sociedade. Como resultado do artigo, apontamos um percurso metodológico com foco na mediação que considera, de um lado, o discurso do objeto enquanto artefato material e do outro, o discurso sobre o objeto enquanto artefato social. Nesse sentido, sugerimos examinar, além da materialidade e visualidade, narrativas e discursos sobre os objetos como perspectiva complementar - o que nos leva a estudar a sua relação com as pessoas dentro do jogo político, e não apenas sobre os artefatos e seus processos de criação e produção. Identificamos que esta abordagem pode ser especialmente útil para pesquisar artefatos gráficos políticos sob uma perspectiva do design. 


\section{Métodos}

Foi realizada uma Revisão Sistemática da Literatura (Castro 2001; Conforto, Amaral e Silva, 2011; Obregon, Vanzin e Ulbricht, 2017), a partir de uma revisão bibliográfica preliminar (Gil, 2009). O objetivo foi levantar as abordagens metodológicas e os métodos de pesquisa do campo da História do Design, História do Design Gráfico e Memória Gráfica no Brasil, que utilizassem aportes da Cultura Material e/ou dos estudos de Memória. O procedimento que orientou a RSL foi o Cochrane Handbook (Cochrane, 2017) e a partir da formulação da pergunta de pesquisa (Castro, 2001), foram percorridas as seguintes etapas:

Quadro 1: etapas da RSL

\begin{tabular}{|c|c|c|c|c|}
\hline $\begin{array}{l}\text { Formulação } \\
\text { da pergunta } \\
\text { de pesquisa }\end{array}$ & $\begin{array}{l}\text { Localização e } \\
\text { seleção dos } \\
\text { estudos }\end{array}$ & $\begin{array}{l}\text { Avaliação } \\
\text { crítica dos } \\
\text { estudos }\end{array}$ & $\begin{array}{l}\text { Análise e } \\
\text { apresentação } \\
\text { dos dados }\end{array}$ & $\begin{array}{l}\text { Interpretação } \\
\text { dos dados e } \\
\text { síntese }\end{array}$ \\
\hline $\begin{array}{l}\text { Quais estudos } \\
\text { Material e dos }\end{array}$ & $\begin{array}{l}\text { na área de } \mathrm{H} \\
\mathrm{s} \text { de Memória }\end{array}$ & $\begin{array}{l}\text { o Design o } \\
\text { arte da abo }\end{array}$ & $\begin{array}{l}\text { Gráfica que se } \\
\text { todológica? }\end{array}$ & de tópicos da Cultura \\
\hline
\end{tabular}

Fonte: a autora.

A partir da definição da pergunta e da revisão bibliográfica preliminar, foram definidas palavraschave (português/inglês) que relacionadas entre si, resultaram em 9 strings de busca (quadro 2).

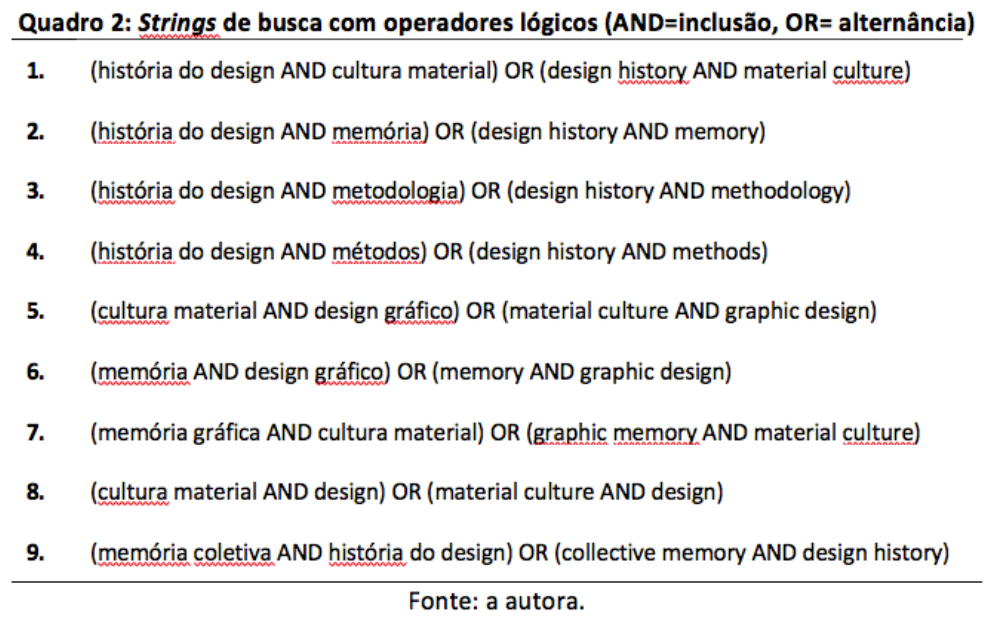

A base de dados escolhida foi o Portal de Periódicos da CAPES e a organização dos dados foi realizada utilizando o software Mendeley. Todas as etapas da RSL, assim como os critérios de filtragem e resultados quantitativos, estão descritos no quadro 3. 
Quadro 3: etapas da RSL e filtragem

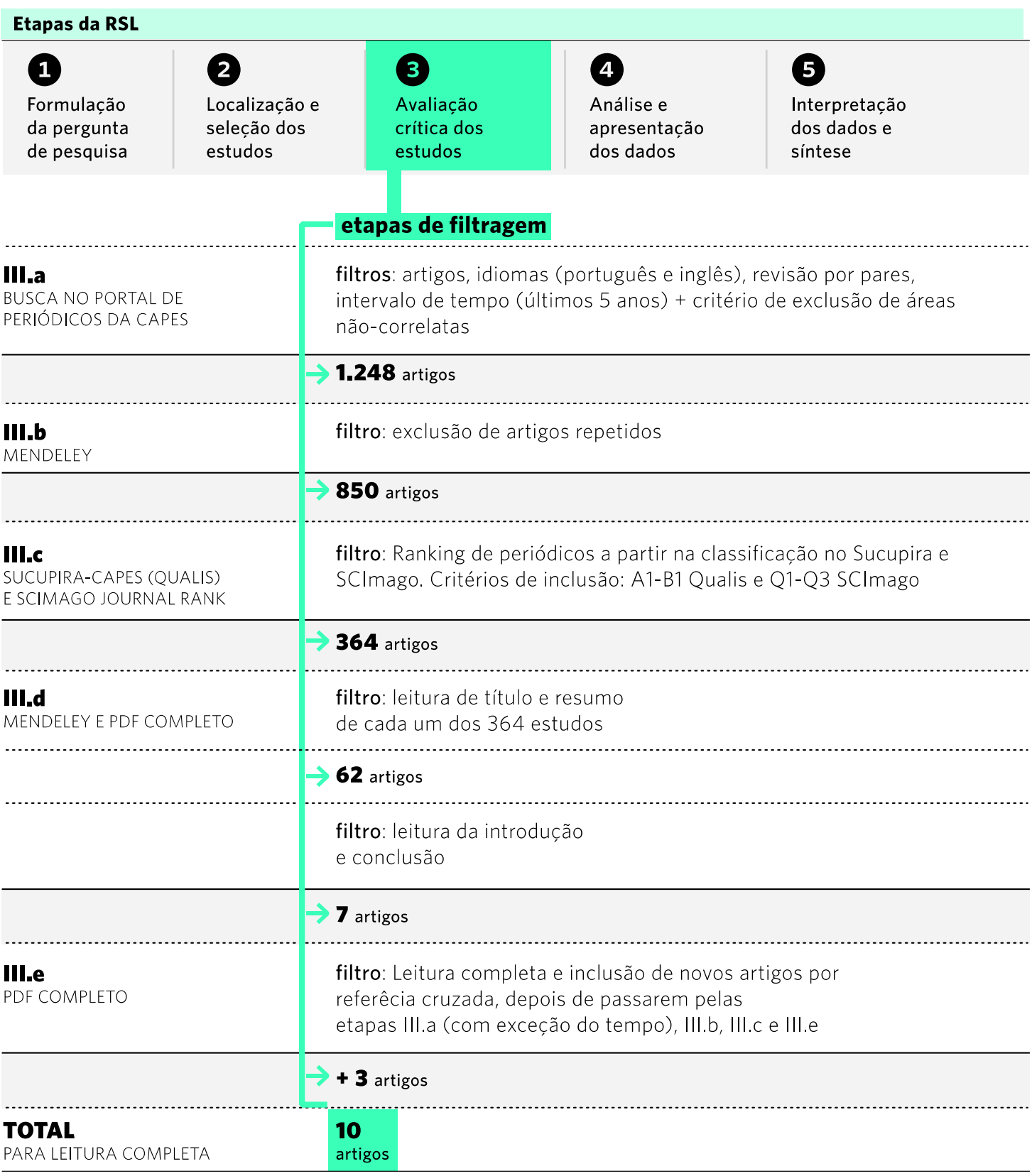

Fonte: a autora.

Já nos quadros 4 e 5, podem ser conferidos os artigos selecionados para leitura completa, que foram classificados em 3 categorias (Callado, 2018): abordagens teórico-metodológicas, métodos aplicados e proposição de método. 
Quadro 4. Lista dos artigos incluídos na rsl, selecionados para leitura completa

\begin{tabular}{|c|c|c|c|c|}
\hline título & autor (es) & fonte da publicação & ano & categoria \\
\hline $\begin{array}{l}\text { Rótulos cromolitográficos } \\
\text { brasileiros: efêmeros, memória } \\
\text { gráfica, cultura material e } \\
\text { identidade nacional }\end{array}$ & $\begin{array}{l}\text { BARROS, } \mathrm{H} \text {. } \\
\text { et al. }\end{array}$ & $\begin{array}{l}\text { Brazilian Journal of } \\
\text { Information } \\
\text { Design, v.13, n. 3, } \\
\text { p. } 321-333\end{array}$ & 2016 & Método aplicado \\
\hline $\begin{array}{l}\text { Conjunto metodológico para } \\
\text { pesquisa em história do design } \\
\text { a partir de materiais impressos }\end{array}$ & $\begin{array}{l}\text { CAMPOS, } \\
\text { A.; } \\
\text { FONSECA, } \\
\text { L.; GOMES, } \\
\text { D. }\end{array}$ & $\begin{array}{l}\text { Brazilian Journal of } \\
\text { Information } \\
\text { Design, v.13, n. 2, } \\
\text { p. 143-161 }\end{array}$ & 2016 & Proposição de método \\
\hline $\begin{array}{l}\text { Gênero e cultura material: } \\
\text { a dimensão política dos } \\
\text { artefatos cotidianos }\end{array}$ & $\begin{array}{l}\text { DOS } \\
\text { SANTOS, } \\
\text { M. }\end{array}$ & $\begin{array}{l}\text { Revista Estudos } \\
\text { Feministas, v.26, n. } \\
\text { 1, p. } 1-8\end{array}$ & 2018 & $\begin{array}{l}\text { Abordagem teórico- } \\
\text { metodológica }\end{array}$ \\
\hline $\begin{array}{l}\text { Introduction to Methodology: } \\
\text { Virtual Special Issue for the } \\
\text { Journal of Design History } 2018\end{array}$ & $\begin{array}{l}\text { HUPPATZ, } \\
\text { D. }\end{array}$ & $\begin{array}{l}\text { Journal of Design } \\
\text { History, v.33, n. 1, } \\
\text { p. e25-e40 }\end{array}$ & 2021 & $\begin{array}{l}\text { Abordagens teórico- } \\
\text { metodológicas }\end{array}$ \\
\hline $\begin{array}{l}\text { Political Murals as Reflection of } \\
\text { Cultural and Ideological } \\
\text { Identity: The Case of Istanul's } \\
\text { Slum Neighbourhoods }\end{array}$ & $\begin{array}{l}\text { ROCKMAN, } \\
\text { S. et al. }\end{array}$ & $\begin{array}{l}\text { Design and } \\
\text { Culture, v.12, n. 1, } \\
319-330\end{array}$ & 2016 & Método aplicado \\
\hline $\begin{array}{l}\text { "Even Our 2nd Class Cars are } \\
\text { More Comfortable Than Motor } \\
\text { Buses!": An Analysis of } \\
\text { Victorian Railway Posters } \\
\text { Between the Wars }\end{array}$ & SYMES, C. & $\begin{array}{l}\text { Journal of } \\
\text { Australian Studies, } \\
\text { v.40, n. 3, 353-371 }\end{array}$ & 2016 & Método aplicado \\
\hline $\begin{array}{l}\text { Creating Life: "America's Most } \\
\text { Potent Editorial Force" }\end{array}$ & WEBB, $\mathrm{S}$. & $\begin{array}{l}\text { Journalism \& } \\
\text { communication } \\
\text { monographs, v.18, } \\
\text { n. 2, 55-108 }\end{array}$ & 2016 & Método aplicado \\
\hline
\end{tabular}

Quadro 5. Lista dos artigos incluídos por referência cruzada, selecionados para leitura completa

\begin{tabular}{|l|l|l|l|l|}
\hline título & autor (es) & fonte da publicação & ano & categoria \\
\hline $\begin{array}{l}\text { Some Futures for Design } \\
\text { History? }\end{array}$ & DILNOT, C. & $\begin{array}{l}\text { Journal of Design } \\
\text { History, v.22, n. 4, } \\
\text { p. 377-394 }\end{array}$ & 2009 & $\begin{array}{l}\text { Teoria, Abordagens } \\
\text { teórico- } \\
\text { metodológicas }\end{array}$ \\
\hline $\begin{array}{l}\text { Why design history? } \\
\text { A multi-national perspective } \\
\text { on the state and purpose } \\
\text { of the field }\end{array}$ & HUPPATZ, & $\begin{array}{l}\text { Arts \& Humanities } \\
\text { in Higher } \\
\text { Education, v.12, n. } \\
\text { D-3, p. 310-330 }\end{array}$ & 2012 & $\begin{array}{l}\text { Abordagem teórico- } \\
\text { metodológica }\end{array}$ \\
\hline $\begin{array}{l}\text { The Production-Consumption- } \\
\text { Mediation Paradigm }\end{array}$ & LEES- & $\begin{array}{l}\text { Journal of Design } \\
\text { History, v.22, n. 4, } \\
\text { p. 351-376 }\end{array}$ & 2009 & $\begin{array}{l}\text { Abordagem teórico- } \\
\text { metodológica, } \\
\text { historiografia }\end{array}$ \\
\hline
\end{tabular}

Fonte: A autora.

A RSL pôde fornecer uma visão ampla e historiográfica sobre metodologias utilizadas na área da História do Design. Entre os estudos, Huppatz (2021) e Lees-Maffei (2009) identificaram correntes mais frequentes. Neste artigo, iremos considerar a classificação de Lees-Maffei (2009) a partir de seu Paradigma Produção-consumo-mediação - o que contribuiu na formatação e validação da abordagem metodológica que será discutida a seguir. 


\section{Paradigma Produção-consumo-mediação e Cultura Material}

Lees-Maffei (2009) faz uma historiografia da História do Design, revisando abordagens usadas no Reino Unido por pesquisadores entre 1979 e 2009. A autora propõe então o paradigma ProduçãoConsumo-Mediação (PCM), onde reconhece três ênfases principais nos estudos da área: produção, consumo e mediação.

A ênfase na produção corresponde ao foco de pesquisa em objetos, processos produtivos e autores, que é predominante desde as primeiras pesquisas na área (Huppatz, 2021; Lees-Maffei, 2009). Na década de 1980, Dilnot $(1984$, p.15) reivindicou um método de abordagem para além da exaltação de artefatos icônicos e designers: "[...] a história do design enfatiza designers individuais. Explícita ou implicitamente, eles são o foco da maior parte da história do design escrita e ensinada hoje".

A segunda ênfase do paradigma PCM se refere ao consumo e abrange a perspectiva do usuário e processos de uso e consumo. Para Lees-Maffei (2009, p.19), essa corrente “[...] continua atraente para historiadores do design que buscam uma compreensão mais holística do design do que aquela permitida por uma ênfase no design profissional e seus fabricantes".

A autora propõe a mediação como uma terceira corrente na História do Design, através de um olhar centrado na função do objeto de design enquanto dispositivo mediador entre os indivíduos e a sociedade: "o foco da mediação permite o reconhecimento que o design é muito mais do que o objeto; é uma teia complexa de práticas e discursos circundantes" (idem, p. 31). Isso não significa um distanciamento do objeto enquanto uma unidade de estudo, mas de assumir uma perspectiva híbrida, considerando a mediação como uma ênfase adicional, complementar e enriquecedora quando somada a uma análise baseada nos objetos e/ou no consumo.

A partir de Igor Kopytoff (1986), Lees-Maffey (2009) parte do pressuposto que os objetos têm histórias de recepção próprias, nem sempre compatíveis com a intenção autoral. Dentro do paradigma PCM, ela identifica três objetos de estudo: (1) o papel dos canais como revistas, televisão, literatura corporativa, etc na mediação entre produtores e consumidores, auxiliando na formação de práticas de consumo e ideias sobre design; (2) o papel dos canais de mediação em si como bens projetados e que podem ser considerados dispositivos mediadores; e (3) objetos/artefatos projetados que fazem a mediação entre o produtor e o consumidor e também são usados como mediadores das relações entre os indivíduos.

O foco na mediação reúne "[...] questões de produção e consumo, mas não através da investigação de intenções projetuais ou de práticas reais de consumo, mas sim através da análise do significado 
cultural e social dos objetos projetados, espaços e processos para revelar ideias e ideais compartilhados" (ibid).

Entretanto, Lees-Maffei (2009) apresenta a mediação num nível teórico, mas não aponta em termos práticos, métodos ou modelos aplicados. Nesse sentido, relacionamos o paradigma PCM com autores do campo da Cultura Material através de um diálogo com Miller (1998), Meneses $(1998,2003)$ e Woodward (2007).

Woodward (2007) define a Cultura material como um campo de estudos que engloba pesquisas sobre os objetos, seus usos e significados e que possibilita uma abordagem multidisciplinar das relações homem-objeto.

Pensando nos significados culturais e sociais atribuídos aos objetos, Miller (1998, p.10) aponta que "[...] ao nos determos nas qualidades materiais e sensuais mais mundanas do objeto, somos capazes de desvendar as conexões mais sutis com vidas e valores culturais que são objetificados por meio dessas formas, em parte, por causa das qualidades particulares que possuem". É como dizer que os objetos têm a capacidade de significar algo e, também, de estabelecer significados sociais em nome das pessoas - um dos pressupostos da Cultura Material (Woodward, 2007). Entretanto, isso pode levar a uma fetichização dos objetos, que consiste "[...] no deslocamento de sentidos das relações sociais - onde eles são efetivamente gerados - para os artefatos, criando-se a ilusão de sua autonomia e naturalidade. Por certo, tais atributos são historicamente selecionados e mobilizados pelas sociedades e grupos nas operações de produção e consumo de sentido. Por isso, seria vão buscar nos objetos o sentido dos objetos" (Meneses, 1998, p. 91).

Dentro das abordagens da Cultura Material, Miller (1998) destaca um caráter único, que é o de permanecer focado no objeto que está sendo investigado, para evitar uma fetichização da forma material e aponta alguns caminhos. Um deles seria resistir a uma contextualização imediata no domínio do objeto de estudo e começar olhando para sua relação com outros domínios materiais.

No entendimento de cultura material como um fenômeno histórico e fonte documental para sua compreensão, podemos pensar que os artefatos gráficos políticos são, de maneira mais ampla, documentos históricos e como tais, suportes físicos de informação histórica (Meneses, 1998).

Contudo, são objetos materiais que exercem, desde antes de sua produção e fundamentalmente depois, um papel mediador propiciando um diálogo entre o indivíduo, as causas emergentes, e a sociedade. Corroborando com Lees-Maffei (2009), Woodward (2007) observa que os objetos são um elo crucial entre a estrutura social e o ator individual. Podemos ainda pensar que esse 
processo de mediação é uma ação política, que pode fazer o objeto existir mesmo antes de sua materialidade, ainda que de forma latente. E que continua para além da veiculação do artefato, identificada por Meneses (2003, p.15-16) como a "ação das imagens, que complementaria o circuito da produção, circulação e da apropriação".

Sobre considerar duas perspectivas numa análise de artefatos, Meneses (1998, p. 91) afirma que:

\author{
"Os traços materialmente inscritos nos artefatos orientam leituras que \\ permitem inferências diretas e imediatas sobre um sem-número de esferas \\ de fenômenos. Assim, a matéria prima, seu processamento e técnicas de \\ fabricação, bem como a morfologia do artefato, os sinais de uso, os indícios \\ de diversas durações, e assim por diante, selam, no objeto, informações \\ materialmente observáveis sobre a natureza e propriedades dos materiais, [...], \\ os aspectos funcionais e semânticos [...] Mas, como se trata de inferência, há \\ necessidade, não apenas de uma lógica teórica, mas ainda do suporte de \\ informação externa ao artefato. [...] Daí a importância da narrativa e dos \\ discursos sobre o objeto para se inferir o discurso do objeto."
}

Neste ponto, evidencia-se a relevância de duas vozes complementares - a do pesquisador e a do ator social que conviveu com os artefatos em seu espaço-tempo. Woodward (2007) aponta que, ao se estudar cultura material, é preciso considerar esses dois pontos de vista. Mas faz ponderações sobre a leitura analítica já que não se trata apenas de indivíduos intuindo sobre o que os objetos podem significar, mas de "indivíduos lendo objetos em relação a outros indivíduos" (idem, p. 4). Para o analista, o objeto pode ser tornado "todo-poderoso, perfeitamente compreensível e historicamente crucial no curso de qualquer reflexão" (ibid). Mas quando a voz do usuário é introduzida, o que antes era estabelecido por medida analítica, abre espaço para práticas e interpretações múltiplas.

\title{
4. Resultados
}

A partir das discussões aqui apresentadas, propomos uma abordagem interdisciplinar híbrida para o estudo de artefatos gráficos políticos, dentro do campo da História do design, a partir de contribuições da Cultura Material (Miller, 1998; Meneses, 1998; Woodward (2007) com foco no papel dos artefatos enquanto dispositivos mediadores (Lees-Maffei, 2009 e 2016; Lees-Maffei e Houze, 2010). 
Como exemplo, trazemos o cartaz abaixo, criado pelo designer e arquiteto Petrônio Cunha e pelo artista plástico Luciano Pinheiro, cujo título é uma mensagem política: Liberdade se conquista. Foi produzido em 1978, na época das campanhas em favor da Anistia, para divulgar uma exposição coletiva realizada para angariar fundos para a candidatura de Jarbas Vasconcelos a senador pelo MDB.

Figura 1. Cartaz pró-candidatura de Jarbas Vasconcelos. Petrônio Cunha e Luciano Pinheiro, 1978.

Fonte: Acervo Fundação Joaquim Nabuco.

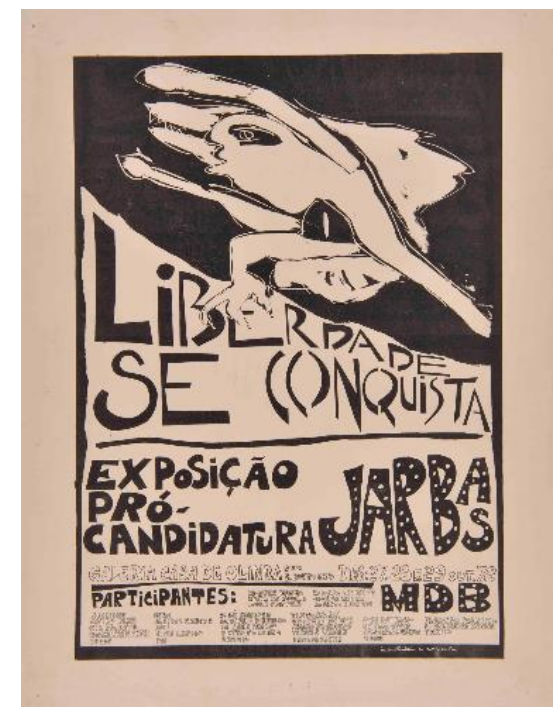

O próprio cartaz pode ser entendido como resultado material de uma ação política, que para acontecer precisa que o artefato seja veiculado. E a partir de sua veiculação, pode favorecer a adesão popular a causas como as campanhas em favor da Anistia, conquistar o eleitorado ou aumentar engajamento nas campanhas eleitorais, por exemplo. Por outro lado, se o cartaz for criado, produzido e engavetado, a ação política não ocorre, já que ele não exerce o seu papel mediador.

Podemos pensar que a mediação acontece através do discurso do artefato (material, visual, simbólico e de conteúdo/informacional), e também através dos discursos sobre o artefato (narrativas a partir da recepção, do uso e da convivência com as pessoas). Juntos, esses dois discursos se complementam e propiciam um diálogo entre indivíduos, questões emergentes e a sociedade, dentro do jogo político.

A partir da materialidade, podemos colher informações sobre processos produtivos, uso de materiais e etc (Menezes, 1998). Como também sobre aspectos visuais, iconográficos, simbólicos 
e informacionais, para inferir o discurso do objeto enquanto artefato material. Entrevistas com autores e produtores também podem auxiliar no entendimento desse discurso, através da apuração de suas motivações, reivindicações e causas políticas/ideológicas/partidárias.

Já o discurso sobre o objeto enquanto artefato social, pode ser investigado através de relatos de memória, como entrevistas e/ou estudos de campo (Gil, 2009) com pessoas que conviveram com os artefatos em seu espaço/tempo (Woodward, 2007) e que não estiveram envolvidas diretamente em sua criação/produção como militantes de partidos, eleitores ou políticos. Também por pesquisas documentais em jornais e periódicos contemporâneos aos artefatos, a partir de matérias que podem conter entrevistas e depoimentos, ou até a descrição do fenômeno/ações políticas.

A partir de tal abordagem, elaboramos uma proposta preliminar de percurso metodológico, que pode ser útil para estudar artefatos gráficos políticos dentro de um contexto histórico. De maneira mais esquemática, a ideia é adotar duas perspectivas de análise complementares, com ênfase na mediação, a seguir:

PERSPECTIVA 1

\section{discurso do objeto enquanto artefato material}

Investigação do discurso do artefato e de sua materialidade

$---$

\section{ARTEFATOS:}

> Observações sistemáticas da materialidade e visualidade: processos produtivos, sinas de uso, técnicas e elementos visuais, iconográficos e simbólicos ligados ao contexto político.

> Observações e coleta de aspectos informacionais: conteúdo/mensagem textual e pictórica/imagética.

AUTORES/PRODUTORES DE ARTEFATOS: $>$ Entrevistas semiestruturadas (Gil, 2009): apuração das motivações políticas, reivindicações/causas e filiações políticas/ ideológicas/partidárias.
PERSPECTIVA 2

\section{discurso sobre o objeto enquanto artefato social}

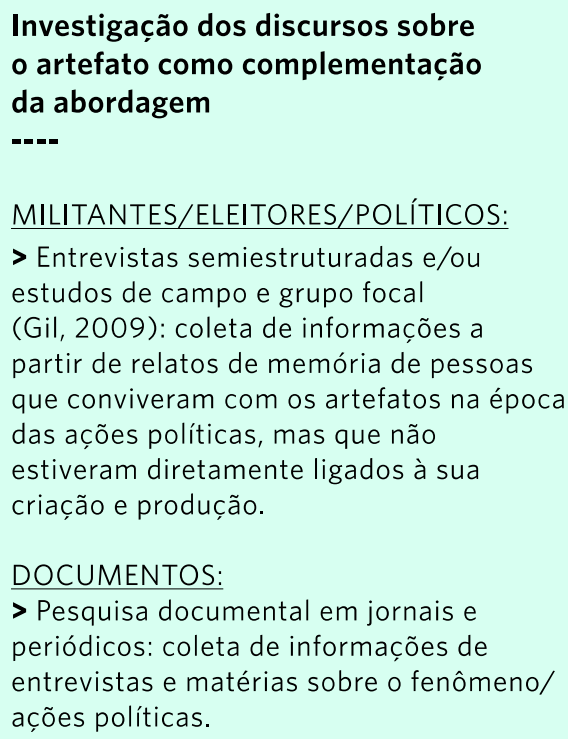
periódicos: coleta de informações de entrevistas e matérias sobre o fenômeno/ ações políticas. 


\section{Conclusão}

Como vimos ao longo deste artigo, através de uma revisão bibliográfica preliminar e de uma Revisão Sistemática da Literatura, delineamos o estado da arte de metodologias na área da História do Design e identificamos 3 correntes metodológicas mais frequentes. Relacionamos o paradigma Produção-consumo-mediação de Lees-Maffei (2009) com contribuições da Cultura Material através de Meneses (1998,2003), Miller (1998) e Woodward (2007).

Consideramos que a dimensão política pressupõe um diálogo que demanda um olhar para o papel dos artefatos dentro do jogo político. Isso nos levou a considerar não apenas a esfera de criação e produção, mas também de veiculação e relação com as pessoas, com foco no papel dos artefatos como dispositivos mediadores. Com base nas discussões apresentadas, formatamos uma proposta de abordagem metodológica para a pesquisa de doutorado "Design Político em Pernambuco: artefatos gráficos e a redemocratização (1979-1989)". Como desdobramento do artigo, futuramente pretendemos detalhar o percurso metodológico e buscar métodos que auxiliem na investigação e análise dos dados. E que possam nos levar a entender também a especificidade do objeto material e sua relação com outros domínios materiais (Miller, 1998). Por fim, esperamos que este estudo e as discussões aqui iniciadas, possam contribuir para futuras pesquisas no campo da História do Design que investiguem artefatos gráficos ligados à política. 


\section{Referências}

BARROS, H; Lessa, W; Lima, E. et al. Rótulos cromolitográficos brasileiros: efêmeros, memória gráfica, cultura material e identidade nacional. São Paulo: Brazilian Journal of Information Design, Infodesign, v. 13, n. 3, p. 199$213,2016$.

BIOLCHINI, J.C.A., Scientific research ontology to support systematic review in software engineering. Advanced Engineering Informatics, v.21, n.2, p.133 - 151, 2007. Naify, 2005

R (Org.). O design brasileiro antes do design: aspectos da história gráfica, 1870-1960. São Paulo: Cosac

CALLADO, Alessandra. Levantamento de requisitos por meio da análise da atividade e da tarefa para sistemas digitais. (Tese não publicada). Programa de pós-graduação em design - PPGD, Universidade Federal de Pernambuco (UFPE), 2018.

CASTRO A.A. Revisão sistemática e meta-análise [texto na Internet]. Disponível em: http://www.metodologia.org/ meta1.PDF. Acesso em: 01 jul. 2021.

COCHRANE COLLABORATION, disponível em: (http://handbook.cochrane.org/). Acessado em abril de 2021.

CONFORTO, C. E.; CALADO, D. A.; DA SILVA, S. L. Roteiro para revisão bibliográfica sistemática: aplicação no desenvolvimento de produtos e gerenciamento de projetos. In Instituto de Gestão de Desenvolvimento do Produto, Congresso Brasileiro de Gestao de Desenvolvimento de Produto-CBGDP (12 p.). Porto Alegre: IBGDP, 2011.

DILNOT, C. The State of Design History Part II: Problems and Possibilities. Design Issues, v. 1, n. 2, p. 3-20, 1984.

FARIAS, P. L. On graphic memory as a strategy for design history. In: International Committee for Design History and Design Studies, Aveiro, Portugal, 2014.

P. L., \& Braga, M. C. Dez ensaios sobre memória gráfica. São Paulo: Blücher, 2018.

FONSECA, L. P., CAMPOS, A. P., \& GOMES, D. D. Conjunto Metodológico para Pesquisa em História do Design a partir de Materiais Impresssos. InfoDesign - Revista Brasileira De Design Da Informação, v. 13, n. 2, p. 143-161, 2016. https://doi.org/10.51358/id.v13i2.481

FRY, T., Design History Australia: A Source Text in Methods and Resources. Sydney: Hale \& Iremonger and the Power Institute of Fine Arts, 1988.

GREEN S, HIGGINS J. P. T., et al. Introduction. In: HIGGINS J. P. T., GREEN S., (Orgs.). Cochrane Handbook for Systematic Reviews of Interventions Version 5.0.1 updated March 2011 [Internet]. Melbourne: The Cochrane Collaboration, 2011. Disponível em: www.cochrane-handbook.org. Acesso em: mar. 2021.

HUPPATZ, D. Introduction to methodology: Virtual special issue for the journal of design history 2018. Journal of Design History. Oxford: University Press, v. 33, p. e25-e40, 2021.

KOPYTOFF, I. The Cultural Biography of Things: Commoditization as Process, in A. Appadurai, ed., The Social Life of Things: Commodities in Cultural Perspective. Cambridge: Cambridge University Press, p. 64 - 91, 1986.

LEES-MAFFEI G. The production-consumption-mediation paradigm. Journal of Design History, v. 22, n. 4, p. 351$376,2009$.

, G., HOUZE, Rebecca (Orgs.). The design history reader. Oxford an New York: Berg, 2010.

G. Design History: The State of the Art. Caa Reviews, 2016.

LESCHKO, N. et al. Memória Gráfica Brasileira: notícias de um campo em construção. In: Anais do 110 Congresso Brasileiro de Pesquisa e Desenvolvimento em Design, 2014, Gramado. V. 1, n. 4, p. 791-803, São Paulo: Editora Edgard Blücher, 2014.

MARTINS, Fernanda de O., LIMA Edna Lúcia C., LIMA, Guilherme C. Análise de duas propostas metodológicas para a pesquisa em História do Design Gráfico. In: C. G. SPINILLO; L. M. FADEL; V. T. SOUTO; T. B. P. SILVA \& R. J. 
CAMARA (Eds.). Anais [Oral] do 7o Congresso Internacional de Design da Informação/Proceedings [Oral] of the 7th Information Design International Conference | CIDI 2015 [Blucher Design Proceedings, num.2, vol.2]. São Paulo: Blucher, 2015.

MENESES, Ulpiano T. Bezerra de. Memória e cultura material: documentos pessoais no espaço público. Estudos Históricos, Rio de Janeiro, v. 11, n.21, 1998.

, Ulpiano T. Bezerra de. Fontes visuais, cultura visual, História visual. Balanço provisório, propostas cautelares. Revista Brasileira de História, São Paulo, v. 23, n.45, p. 11-36, 2003.

MILLER, Daniel (ed.). Material cultures: Why some things matter. Londres, UCL, 1998.

Mendeley. Disponível em: http://www.mendeley.com/. Acesso em: 15 abr. 2021.

OBREGON, R.; VANZIN, T.; ULBRICHT, V. Recomendações para Ambiente Virtual de Aprendizagem Inclusivo na perspectiva do Padrão Arquetípico de Alteridade. São Paulo: Ed. Pimenta Cultural, 2015.

WEBB, S. Creating Life: "America's Most Potent Editorial Force". Journalism \& communication monographs, v. 18, n. 2, p. 55-108, 2016.

WOODWARD, Ian. Understanding Material Culture. Londres: SAGE, 2007. 